Statistics in the Twenty-First Century: Special Volume

In Honour of Distinguished Professor Dr. Mir Masoom Ali

On the Occasion of his 75th Birthday Anniversary

PJSOR, Vol. 8, No. 3, pages 531-535, July 2012

\title{
On Introducing Asymmetry into Circular Distributions
}

\author{
Dale Umbach \\ Ball State University \\ dumbach@bsu.edu \\ S. Rao Jammalamadaka \\ University of California \\ Santa Barbara \\ rao@pstat.ucsb.edu
}

\begin{abstract}
We give a brief history of the results which led to the introduction of asymmetry into symmetric circular distributions. This is followed by the presentation of another method of introducing asymmetry. Some properties of the induced distributions are studied. Finally, this new distribution is shown to be a reasonable fit to the Jander ant data as presented in Fisher (1993).
\end{abstract}

\section{Introduction and Main Result}

Azzalini $(1985,1986)$ introduced a method of skewing a symmetric distribution to produce tractable families of non-symmetric distributions. He showed that with $f$ the density of a symmetric distribution and $G$ being the distribution function of a symmetric distribution that

$$
2 G(\lambda x) f(x)
$$

is a density for each $\lambda$ and that $\lambda$ controls the amount of skew. This generated a large number of papers producing skewed models from many of the well-known symmetric distributions. Ali, Pal, and Woo (2008 and 2009) and Nadarajah and Ali (2005) are good examples of such work.

Later, Azzalini and Capitanio (1999) showed more generally that

$$
2 G(w(x)) f(x)
$$

is a density where $f$ and $G$ are as above and $w$ is odd. Along this line, Umbach and Jammalamadaka (2009) introduced this skewing idea to circular distributions. They showed that with $f$ a symmetric (about 0 ) circular density and $G$ the distribution function of a symmetric (about 0 ) circular density and $w$ odd and periodic with $|w(\theta)| \leq \pi$ that

$$
2 f(\theta) G(w(\theta))
$$

is a circular density with $G(-\pi)=0$. This typically results in an asymmetric distribution. One can introduce various parameters by judicious choices of $G$ and $w$. 
In this work, we introduce a different method of introducing asymmetry into circular distributions. In particular, we show that

$$
p(\theta ; \gamma)=\frac{2 f(\theta)(1+(\gamma-1) G(w(\theta)))}{\gamma+1}
$$

is a circular density for each $\gamma \geq 0$, with $f, G$, and $w$ as above. Results and interpretation concerning the introduction of the parameter $\gamma$ will be presented.

Theorem: Suppose that $G$ is the distribution function of a symmetric (about 0 ), circular distribution and that $f$ is the density of a symmetric (about 0 ), circular distribution, and that $w$ is an odd function with $|w(\theta)| \leq \pi$ and $\gamma \geq 0$, then $p(\theta ; \gamma)$ from (4) is a circular density.

The proof is immediate from (3) and noting that $(1+(\gamma-1) G(w(\theta)) \geq(1-G(w(\theta)) \geq 0$.

Figure 1 gives some indication for the amount of asymmetry present in the density $p$. The central symmetric density is that of the von Mises distribution

$$
f(\theta)=\frac{e^{\kappa \cos (\theta)}}{2 \pi I_{0}(\kappa)},
$$

with $I_{0}(\kappa)$ being the modified Bessel function of the first kind with $\kappa=3$. The densities graphed are for $\gamma=1,0.1,2$, and 10 and $G(\theta)=(\theta+\pi) /(2 \pi)$ (uniform distribution) and $w(\theta)=\pi \sin (\theta)$.

\section{Properties of the Distribution}

To develop some properties of the distribution $p$ let us write

$$
p(\theta ; \gamma)=\frac{2}{\gamma+1} f(\theta)+\frac{\gamma-1}{\gamma+1} 2 f(\theta) G(w(\theta))
$$

Thus, we can express $p$ as quasi-mixture of the symmetric central density $f$ and the distribution introduced by Umbach and Jammalamadaka (2009) in (3). Note that the coefficients do add to 1 , but $(\gamma-1) /(\gamma+1)$ can be negative.

However, we can express $p$ as a proper mixture of different distributions. It is easily seen that

$$
\begin{aligned}
& \text { (4) } \left.=\frac{1}{\gamma+1}\{2 f(\theta)+\gamma 2 f(\theta) G(w(\theta))-2 f(\theta)) G(w(\theta))\right\} \\
& =\frac{1}{\gamma+1} 2 f(\theta) \bar{G}(w(\theta))+\frac{\gamma}{\gamma+1} 2 f(\theta) G(w(\theta)),
\end{aligned}
$$

with $\bar{G}(\theta)=1-G(\theta)$.

Next we show that $f_{1}(\theta)=2 f(\theta) \bar{G}(w(\theta))$ is also a circular density under the conditions that yielded (3). Note that the symmetry of $G$ implies $G(-\theta)=\bar{G}(\theta)$. 


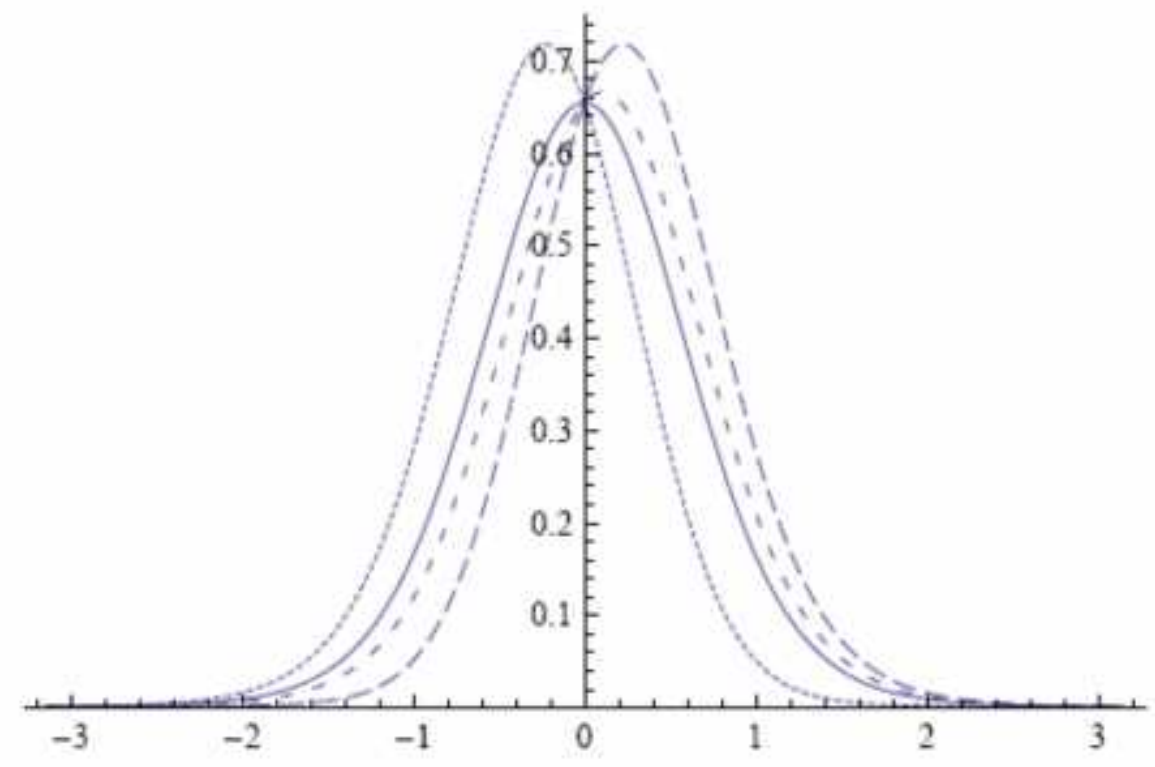

Figure 1: Graphs of the skewed von Mises distribution with $\kappa=3$ and $\gamma=1$ (the von Mises itself)(solid line), $\gamma=0.1$ (dotted line), $\gamma=2$ (sparse dashing), and $\gamma=10$ (dense dashing).

Noting that $w$ will be odd, periodic and bounded by $\pi$ precisely when $-w$ is odd, periodic and bounded by $\pi$, we see that $p$ is a proper mixture of the distributions in (7).

In Umbach and Jammalamadaka (2010), they showed that if $h$ is even and periodic that

$$
\int_{-\pi}^{\pi} h(\theta) f(\theta) d \theta=\int_{-\pi}^{\pi} h(\theta) 2 f(\theta) G(w(\theta)) d \theta .
$$

We note that this property of expectations of even functions is shared in the linear case by all distributions that are skewed in the Azzalini style. Since $p$ is a linear combination of the densities in (6) we see that the common value of the integrals in (6) is also the value of

$$
\int_{-\pi}^{\pi} h(\theta) p(\theta, \gamma) d \theta
$$

for all $\gamma>0$.

One can apply the "odd function" results of Umbach and Jammalamadaka (2010) as well. In particular, note that for $h$ odd and periodic, we have

$$
\int_{-\pi}^{\pi} h(\theta) p(\theta, \gamma) d \theta=\frac{\gamma-1}{\gamma+1} \int_{-\pi}^{\pi} h(\theta) 2 f(\theta) G(w(\theta)) d \theta .
$$

In the next section, we apply these results to trigonometric moments.

\section{Trigonometric moments}

For a circular random variable $\Theta$, the $p^{\text {th }}$ trigonometric moment $\varphi_{p}$, is defined by 


$$
\varphi_{p}=E\left(e^{i p \Theta}\right)=E(\cos p \Theta)+i E(\sin p \Theta)=\alpha_{p}+i \beta_{p} .
$$

Let $\rho_{p}$ be the length of $\varphi_{p}$ and if $\varphi_{p}$ is not 0 , let $\mu_{p}$ be its direction. $\mu_{1}$ is typically referred to as the mean (or preferred) direction. We shall compare and contrast the moments of the distributions presented herein. To streamline the results, we shall refer to quantities calculated for the cental symmetric distribution, $f$, with the superscript ${ }^{o}$, as $\varphi_{p}^{o}$, for example. We shall refer to quantities calculated for the distribution in (3) with the superscript ', as $\mu_{p}^{\prime}$, for example. We shall use the superscript * in relation to quantities calculated for the distribution $p$ in (5) and will use the superscript ${ }^{*}$ in relation to the folded distribution $2 f(\theta)$ for $0 \leq \theta \leq \pi$. Umbach and Jammalamadaka (2010) showed that

$$
0=\beta_{1}^{o} \leq \beta_{1}^{\prime} \leq \beta_{1}^{*} .
$$

Noting that $\beta_{1}^{\star}=((\gamma-1) /(\gamma+1)) \beta_{1}^{*}$, we see that

$$
\begin{aligned}
& 0=\beta_{1}^{o} \leq \beta_{1}^{\star} \leq \beta_{1}^{\prime} \leq \beta_{1}^{*} \text { for } \gamma \geq 1 \text { and } \\
& \beta_{1}^{\star} \leq 0=\beta_{1}^{o} \leq \beta_{1}^{\prime} \leq \beta_{1}^{*} \text { for } 0<\gamma \leq 1 .
\end{aligned}
$$

The "even function" results above directly yield

$$
\alpha_{p}^{o}=\alpha_{p}^{\star}=\alpha_{p}^{\prime}=\alpha_{p}^{*} \text { forp }= \pm 1, \pm 2, \ldots,
$$

and thus

$$
0<\rho_{1}^{o} \leq \rho_{1}^{\star} \leq \rho_{1}^{\prime} \leq \rho_{1}^{*}
$$

Combining (11) and (12), we see that

$$
\begin{aligned}
& 0=\mu_{1}^{o} \leq \mu_{1}^{\star} \leq \mu_{1}^{\prime} \leq \mu_{1}^{*} \text { for } \gamma \geq 1 \text { and } \\
& \mu_{1}^{\star} \leq 0=\mu_{1}^{o} \leq \mu_{1}^{\prime} \leq \mu_{1}^{*} \text { for } 0<\gamma \leq 1 .
\end{aligned}
$$

\section{An Application}

Rudolf Jander's experiments concerning the direction of ants in response to a stimulus has long provided some interesting problems in modeling of circular distributions. See Jander (1957) for an original description of these experiments. In particular, the data set of Example 4.4 on page 60 of Fisher (1993) has generated much interest. Fisher clearly demonstrates that the von Mises distribution does not fit this data very well. We come closer to an appropriate model using an asymmetric model as presented herein.

We will model the distribution with the density

$$
p(\theta ; \mu, \kappa, \gamma)=\frac{e^{\kappa \cos (\theta-\mu)}}{(\gamma+1) 2 \pi I_{0}(\kappa)}(1+\gamma+(\gamma-1) \sin (\theta-\mu)) \text {. }
$$

This density results from the choices of $f$ being the von Mises distribution of (5), $G(\theta)=(\theta+\pi) /(2 \pi)$ (uniform distribution), and $w(\theta)=\pi \sin (\theta)$ and finally introducing a directional parameter $\mu$. 
Mathematica was used to numerically carry out maximization of the likelihood with the following results.

$$
\begin{aligned}
& \hat{\mu}=2.88018=165.053^{\circ} \\
& \hat{\kappa}=1.4361 \\
& \hat{\gamma}=3.19855 .
\end{aligned}
$$

To provide some guidance as to the goodness of the fit, we calculated both the Kuiper and Watson statistics for the model in (14) with the mle's used for the parameters. The value of the Kuiper statistic as described on page 153 of Jammalamadaka and SenGupta (2001) is $V_{100}=2.33717$. The value of the Watson statistic on page 156 of the same text is 0.287414 . Both these indicate a reasonable fit of the model (14) to this data. On the other hand, Fisher (1993, p.84) tried fitting the vonMises model in (5) to this data and found the maximum likelihood estimators to be

$$
\hat{\mu}=3.1957=183.1^{\circ} \text { and } \hat{\kappa}=1.54 \text {. }
$$

After doing formal goodness of fit tests as well as Q-Q plots, he concludes that the vonMises distribution is not a suitable model for this data.

\section{References}

1. Ali, M., Pal, M., and Woo J. (2008), Skewed reflected distributions generated by reflected gamma kernel. Pakistan Journal of Statistics, 24, 77-86.

2. Ali, M., Pal, M., and Woo J. (2009), Skewed reflected distributions generated by the Laplace kernel. Austrian Journal of Statistics, 38, 45-58 .

3. Azzalini, A. (1985), A class of distributions which includes the normal ones, Scandinavian Journal of Statistics, 12 171-178.

4. Azzalini, A. (1986), Further results on a class of distributions which includes the normal ones, Statistica, 46, 199-208.

5. Azzalini, A., and Capitanio, A. (1999), Statistical applications of the multivariate skew-normal distribution. Journal of the Royal Statistical Society, Series B, 61, 579-602.

6. Fisher, N.I. (1993), Statistical Analysis of Circular Data, Cambridge University Press, Melbourne.

7. Jammalamadaka, S. R., and SenGupta, A. (2001), Topics in Circular Statistics, World Scientific, Singapore.

8. Jander, R. (1957), Die optische Richtungsorientierung der roten Waldameise (formica Rufa L.), Zeitschrift für Vergleichende Physiologie, 40, 162-238.

9. Nadarajah, S. and Ali, M. (2005), A skewed truncated $t$ distribution, Mathematical and Computer Modelling, 40, 935-939.

10. Umbach, D. and Jammalamadaka, S. R. (2009), Building asymmetry into circular distributions, Statistics and Probability Letters, 79, 659-663.

11. Umbach, D. and Jammalamadaka, S. R. (2010), Some moment properties of skew-symmetric distributions, Metron, LXVIII, 265-273. 